\title{
SOBRE A APLICAÇÃO DE SISTEMAS INTELIGENTES PARA DIAGNÓSTICO DE FALHAS EM MÁQUINAS DE INDUÇÃO - UMA VISÃO GERAL
}

\author{
Fernanda Maria da Cunha Santos* \\ fcunha@sc.usp.br
}

\author{
Ivan Nunes da Silva* \\ insilva@sc.usp.br
}

\author{
Marcelo Suetake* \\ mclsuetake@gmail.com \\ *Universidade de São Paulo, Escola de Engenharia de São Carlos \\ Departamento de Engenharia Elétrica \\ Av. Trabalhador Sancarlense, 400, Centro, CEP 13566-590
}

São Carlos - SP

\begin{abstract}
On the Application of Intelligent Systems for Fault Diagnosis in Induction Motors

Many researches have been developed in order to design intelligent systems for diagnosing faults in electrical machines. These faults involve since electrical problems, such as short circuit in one phase of the stator, until mechanical problems, such as bearing damage. Among the intelligent systems applied in these situations, we highlight the artificial neural networks, the fuzzy inference systems, the genetic algorithms and the hybrid systems, such as those neuro-fuzzy approaches. This paper describes an overview of the most relevant works in this area, which have obtained promising results from the use of intelligent systems in the processes involved to the fault diagnosis in electrical motors. Its main contribution is in providing several technical aspects in order to address future works in this application field.
\end{abstract}

KEYWORDS: Electrical machines, induction motors, artificial neural network, fuzzy systems, fault diagnosis, intelligent systems.

Artigo submetido em 25/03/2011 (Id.: 1309) Revisado em 19/06/2011, 04/10/2011, 22/11/2011

Aceito sob recomendação do Editor Associado Prof. Jés de Jesus Fiais Cerqueira

\section{RESUMO}

Muitas pesquisas estão sendo desenvolvidas buscando nos sistemas inteligentes soluções para diagnosticar falhas em máquinas elétricas. Estas falhas envolvem desde problemas elétricos, como curto-circuito numa das fases do estator, até problemas mecânicos, como danos nos rolamentos. Dentre os sistemas inteligentes aplicados nesta área, destacam-se as redes neurais artificiais, os sistemas fuzzy, os algoritmos genéticos e os sistemas híbridos, como o neuro-fuzzy. Assim, o objetivo deste artigo é traçar um panorama geral sobre os trabalhos mais relevantes que se beneficiaram dos sistemas inteligentes nas diferentes etapas de análise e diagnóstico de falhas em motores elétricos, cuja principal contribuição está em disponibilizar diversos aspectos técnicos a fim de direcionar futuros trabalhos nesta área de aplicação.

PALAVRAS-CHAVE: Máquinas elétricas, motores de indução, redes neurais artificiais, sistemas fuzzy, identificação e diagnóstico de falhas, sistemas inteligentes.

\section{INTRODUÇÃO}

Sistemas para diagnosticar falhas em motores elétricos, principalmente em motores de indução trifásicos (MIT), estão sendo pesquisados e desenvolvidos por profissio- 
nais da área acadêmica e da indústria. Os objetivos destes estudos são interpretar precocemente a falha incipiente e definir um correto diagnóstico para que não ocorra uma manutenção não-programada e uma parada no processo produtivo. Prevenindo a ocorrência de falhas, pode-se também evitar consequiências como aquecimento excessivo, desbalanceamento da corrente e da tensão, decaimento do torque médio, redução da eficiência e grandes perdas financeiras (Nandi et al., 2005). Em outras palavras, os aumentos da disponibilidade e da confiabilidade das máquinas se tornam as principais razões pela crescente demanda por sistemas de manutenção preditivos e de diagnóstico de faltas automatizados.

De acordo com Bonnett and Yung (2008), as porcentagens de falhas em MIT podem ser distribuídas em: rolamentos $(69 \%)$, enrolamento do estator $(21 \%)$, barra do rotor $(7 \%)$ e o eixo $(3 \%)$. A maneira para detectar estes problemas mecânicos e elétricos pode ser feita direta ou indiretamente por meio da leitura de uma das fases na corrente elétrica de alimentação ou pela tensão elétrica, medidas de temperatura, monitoramento do campo eletromagnético ou por outros parâmetros (Ghate and Dudul, 2010).

Três métodos são utilizados, principalmente, na detecção e diagnóstico de falhas em MITs: técnicas baseadas em modelos, sistemas especialistas e algoritmos de sistemas inteligentes que simulam modelos biológicos. Técnicas baseadas em modelos são muito proeminentes na obtenção de um modelo exato do processo. Em motores de indução, um modelo exato de todo o sistema é de difícil obtenção, além da imprecisão, devido à especificação de valores de componentes, limitações do motor e ocorrência de eventos não-programados (Zidani et al., 2008). Além disso, a execução dos métodos convencionais, baseados em modelos, consome muito tempo nas tarefas computacionais por causa de suas complexidades e pelas grandes quantidades de parâmetros de desempenho (Filippetti et al., 2000).

Os sistemas especialistas, geralmente dedicados a sistemas com uma grande quantidade de dados sobre um determinado processo, e implementados por linguagens de programação específicas, necessitam da existência de um perito para a construção de regras e da árvore de raciocínio (Zidani et al., 2008). A introdução de algoritmos de sistemas inteligentes é um passo em direção a uma maior flexibilidade, pois não são tão dependentes da disponibilização de um modelo, tendo-se também a capacidade de aprendizado a partir da extração de conhecimento sobre situações que envolvem o processo.
Recentemente, os sistemas de diagnóstico de falhas optam por métodos não-invasivos durante a aquisição dos dados e técnicas diferentes estão sendo desenvolvidas nas etapas subseqüentes. Dentre estas, destacam-se os sistemas inteligentes baseados em Redes Neurais Artificiais (RNAs), Lógica Fuzzy (LF) (Zadeh, 1965), Algoritmos Genéticos (AGs) (Goldberg, 1989) e sistemas híbridos. Uma das principais vantagens no uso de sistemas inteligentes é sua implementação com baixa complexidade computacional, pois é capaz de determinar o tipo de uma falha num MIT sem necessitar de modelos matemáticos complexos.

Assim, neste artigo é apresentada uma visão geral sobre a atuação dos diferentes tipos de sistemas inteligentes em quase todas as etapas do processo de diagnóstico de falhas nos motores de indução, tendo-se também o intuito de se identificar ainda os principais problemas em aberto, assim como apontar algumas tendências para pesquisas futuras. Mais especificamente, será descrito aqui quais tipos de sinais que são lidos das máquinas, as técnicas implementadas na extração das características presentes nos sinais, a seleção dos dados e a classificação da falha. Assim sendo, uma vasta bibliografia dos trabalhos mais relevantes são apresentados neste artigo, detalhando-se as metodologias dos que utilizaram sistemas inteligentes, assim como daqueles que aplicaram técnicas convencionais.

O artigo está organizado em seções que relatam, primeiramente (Seção 2), os tipos de sinais lidos do MIT e as respectivas falhas que podem ser interpretadas pelos mesmos. A Seção 3 expõe as formas de acionamento de um MIT, com o intuito de destacar a participação dos inversores no sistema. A Seção 4 descreve as técnicas utilizadas na identificação de falhas geradas pelo estator e, na seqüência (Seção 5) as técnicas de diagnóstico para falhas no rotor são apresentadas. A Seção 6 destaca as falhas mecânicas geradas pelos rolamentos e como identificá-las. A Seção 7 relata as falhas relacionadas à excentricidade. A Seção 8 discorre sobre os problemas ainda em aberto quanto ao diagnóstico de falhas em máquinas de indução, assim como aponta as novas tendências relacionadas às frentes de pesquisa. Por fim, na Seção 9 se destaca alguns relevantes aspectos relacionados à temática do artigo.

\section{TIPOS DE SINAIS EM MIT}

As principais falhas em motores elétricos são originadas por fatores elétricos ou mecânicos, de acordo com as descrições formuladas em Bellini et al. (2008a) e Tallam et al. (2007). Estas falhas podem ser monitoradas por métodos não-invasivos que fazem à leitura de valores 
quantitativos de variáveis, como a vibração, a corrente, a tensão, o torque e a velocidade (Bellini et al., 2008a).

A vibração está relacionada a parâmetros como massa, amortecimento e rigidez. Elementos rolantes e rolamentos dos motores podem gerar fadiga entre os materiais que os envolvem devido à carga submetida sobre eles. Logo, um pequeno desgaste na trajetória ou nos rolamentos gerará vibrações intensas devido aos impactos repetitivos do movimento dos componentes defeituosos, quando estes estiverem numa rápida aceleração. Assim, os sinais de vibração gerados pelos elementos rolantes e rolamentos poderão determinar falhas como descamação, estilhaçamento ou quebra dos rolamentos.

Os sinais de vibração também são usados para detectar a presença de falhas mecânicas nos rolamentos e na barra do rotor (Baccarini et al., 2011), mas os sensores (emissão acústica) exigidos podem ser caros e de difícil instalação, além de serem influenciados por ruídos inerentes ao próprio funcionamento normal da máquina (Bellini et al., 2008b; Frosini and Bassi, 2010). Os sinais elétricos são preferidos em muitas situações em virtude de ser facilmente acessado, confiável e, principalmente, por traduzir diferentes tipos de falhas (Immovilli et al., 2010).

A assinatura da corrente do estator relata problemas como quebras na barra do rotor, excentricidade no entreferro, danos nos rolamentos, anomalias na carga e falhas nos enrolamentos do estator (Bellini et al., 2008a; Frosini and Bassi, 2010). Em outras palavras, os componentes da corrente podem representar mais de uma falha, além de algumas características estar escondidas em ruídos devido às baixas frequências. Logo, torna-se necessária a aplicação de técnicas de processamento de sinais para extração dessas características.

A corrente do estator, combinada com outros parâmetros do motor, são lidos para garantir maior confiabilidade na detecção de algumas falhas. Por exemplo, a corrente e a velocidade do rotor são sugeridos para analisar as condições dos rolamentos e da isolação de enrolamentos dos motores (Goode, 1995b). Entretanto, variações de torque de carga aplicado ao eixo do motor mais a velocidade, também representam parâmetros usados para avaliar as condições de funcionamento do motor de indução trifásico, mediante o ajuste da amplitude e da frequência da tensão de alimentação do motor (Suetake et al., 2010).

O sinal de tensão do estator também é usado para detectar problemas no rotor, principalmente quebras nas barras do rotor. O sinal padrão do suprimento de tensão é analisado e seu ciclo médio é determinado a fim de ser usado como comparação com outros sinais, pois oscilações específicas no ciclo médio aumentam quando há falhas nas barras do rotor (Nemec et al., 2010). Ademais, os sinais de corrente e de tensão do estator são analisados juntamente para detectar falhas nas barras do rotor (Karami et al., 2010b).

Após a captação dos sinais, técnicas de processamento de sinais são executadas a fim de melhorar a relação sinal/ruído e normalizar os dados para isolar a falha de outros fenômenos. Técnicas no domínio tempo-frequência estão sendo amplamente usadas nas investigações, como a Short-Time Fourier Transform (STFT), a distribuição Wigner Ville (WVD), a transformada de Hilbert, a análise de envoltória e a transformada wavelet. Por conseguinte, o uso de sistemas inteligentes baseados em objetos e regras se faz necessário para atuar como o administrador no processo de diagnóstico, pois, a partir dos dados extraídos, aplicar-se-ão suas estratégias determinísticas e heurísticas a fim de abstrair cada falha dos MIT.

\section{MODOS DE ACIONAMENTOS DE MIT}

Os MIT podem ser acionados diretamente pela rede elétrica, cujos sinais são caracterizados como senoidais, ou por inversores de frequência, cujos sinais são nãosenoidais. Os inversores de frequência PWM, composto pelo modo de controle open-loop ou closed-loop, são responsáveis em controlar a frequência da velocidade do rotor.

Os inversores open-loop aparecem como uma fonte controladora de tensão AC para o motor. Já o inversor closed-loop, em princípio, tem um regulador de corrente de alta largura de banda, o que faz ele aparecer para o motor como uma fonte controladora de corrente AC. No entanto, vários dispositivos de controle closed-loop surgem como fontes controladoras de corrente e tensão. Nesta situação, a assinatura da falta estará presente tanto na corrente do motor quanto na tensão, o que aumenta a dificuldade em definir falhas num MIT (Pires et al., 2009).

Métodos convencionais para análise de sinais de MIT são ineficientes ao identificar falhas em motores acionados por inversores. Isto se justifica pelo controlador de realimentação de corrente que mascara a assinatura da falta, ou ainda, pela mudança constante da carga, ou senão, pela produção de componentes de frequências próximos à componente fundamental, os quais indicam a quebra na barra do rotor (Bellini et al., 2008a; Kim et al., 2011). 
Tabela 1: Falhas dos Componentes do Enrolamento do Estator

\begin{tabular}{|l|l|}
\hline Componentes & Tipos de Falhas \\
\hline \multirow{3}{*}{ Condutores } & Mangueiras de arrefecimento \\
\cline { 2 - 2 } & Sub-condutores \\
\cline { 2 - 2 } Isolamento & Barra do condutor \\
\hline \multirow{2}{*}{ Enrolamento Final } & Parede principal de isolamento \\
\cline { 2 - 2 } & Isolação dos sub-condutores \\
\cline { 2 - 2 } & Isolação \\
\cline { 2 - 2 } & Movimento ou Fricção \\
\hline
\end{tabular}

Todavia, diversos trabalhos relatam o aumento da presença de inversores nos sistemas de detecção e diagnóstico de falhas de motores, sendo estes responsáveis pelo fornecimento de variáveis elétricas para o sistema, além de atuarem como mais um componente gerador de falhas. Na literatura, destacam-se o uso de inversores closed-loop ou open-loop, aliados aos modelos matemáticos e às técnicas de processamento de sinais, a fim de identificar falhas no rotor, principalmente as quebras nas barras do rotor (Ayhan et al., 2008; Nemec et al., 2010; Kim et al., 2011; Wolbank et al., 2011) e as falhas no enrolamento do estator (Briz et al., 2008; Cheng et al., 2011). No artigo Lee et al. (2011) os autores não relatam falhas específicas do motor, porém destacam uma metodologia que analisam variáveis de todo o sistema e que podem levar às principais falhas.

Como os métodos tradicionais podem esconder problemas dos MIT acionados por inversores, reforça-se aqui a busca por novos métodos para analisar o estado técnico dos dispositivos elétricos. Dentre estes métodos destacam os sistemas inteligentes, os quais podem ser implementados em controladores ou em aplicações offline do sistema de diagnóstico. Em trabalhos recentes encontram-se as RNA aplicadas na identificação de falhas do rolamento (Onel et al., 2009; Cho et al., 2010), em barras quebradas do rotor (Kaminski et al., 2010) e em falhas no enrolamento do estator (Cho et al., 2010). A LF é utilizada na detecção de curtos-circuitos nos enrolamentos do estator (Rodriguez and Arkkio, 2008) e os sistemas híbridos neuro-fuzzy no diagnóstico de falhas no rotor (Uddin et al., 2010) e no rolamento (Abu-Rub et al., 2010).

\section{FALHAS NO ESTATOR}

Conforme Vas (1993), as falhas originadas pelo enrolamento do estator em máquinas de indução podem ser subdivididas de acordo com seus componentes, como descrito na Tabela 1.
Em um motor de indução de baixa tensão, as falhas do enrolamento do estator podem ser classificadas em: curto-circuito entre espiras de uma mesma bobina, curto-circuito entre bobinas da mesma fase, curtocircuito entre bobinas de diferentes fases, curto-circuito entre uma fase e o terra e circuito aberto em uma fase.

O curto-circuito entre os enrolamentos de cobre causa um fluxo significativo de corrente na bobina levando a uma rápida deteriorização. Esta falha é muito destrutiva, pois envolve a queima do isolamento e fusões localizadas nos condutores de cobre. O monitoramento contínuo da seqüência negativa da corrente do estator é um simples método para detecção desta falha.

Quando o motor está girando, o rotor movimenta na direção da corrente de sequiência positiva, próximo à velocidade síncrona. A corrente de sequiência negativa, com uma rotação de fase oposta à rotação do rotor, gera uma tensão que induz uma corrente substancial no rotor. Essa corrente induzida tem uma frequência de aproximadamente duas vezes a frequência da rede. O efeito pelicular dessa frequência nas barras do rotor causa um significante aumento na resistência do rotor e, consequentemente, uma significativa elevação no aquecimento do rotor.

As seqüências negativas, positivas e zero são usadas para transformar um conjunto genérico de fasores em vetores balanceados. Especificamente, três correntes de linha $\left(\bar{I}_{u}\right),\left(\bar{I}_{v}\right)$ e $\left(\bar{I}_{w}\right)$ são transformadas em três vetores balanceados conhecidos como seqüência positiva $\left(\bar{I}_{p}\right)$, seqüência negativa $\left(\bar{I}_{n}\right)$ e seqüência zero $\left(\bar{I}_{0}\right)$, isto é:

$$
\left[\begin{array}{c}
\bar{I}_{p} \\
\bar{I}_{n} \\
\bar{I}_{0}
\end{array}\right]=\frac{1}{3}\left[\begin{array}{ccc}
1 & \alpha & \alpha^{2} \\
1 & \alpha^{2} & \alpha \\
1 & 1 & 1
\end{array}\right]\left[\begin{array}{l}
\bar{I}_{u} \\
\bar{I}_{v} \\
\bar{I}_{w}
\end{array}\right]
$$

onde $\alpha=e^{j \theta}, \theta=\frac{2 \Pi}{3}$.

Em Kohler et al. (1992), os sinais de tensão e corrente são adquiridos com a finalidade de calcular a seqüência negativa. Visto que o valor dessa seqüência é constante num motor em condições saudáveis, sugere-se então que um alarme seja disparado se o desvio da seqüência for superior a $6 \%$, levando-se ao desligamento da máquina antes que aumente as falhas detectadas.

Muitos trabalhos reconhecem a corrente de seqüência negativa como procedimento de diagnóstico que distingue diferentes fenômenos dos motores (Kohler et al., 1992; Bellini et al., 2000). Dentre esses fenômenos destacam a identificação de assimetria do estator e curtocircuitos nos enrolamentos, principalmente quando estes ocorrem em poucas espiras. Um procedimento de diagnóstico eficiente deve distinguir entre uma seqüência ne- 
gativa, causada pelo curto-circuito, daquelas seqüências geradas por tensões desbalanceadas, saturação, assimetrias nos enrolamentos e excentricidades.

A abordagem do vetor de Park é outro método usado com sucesso para detectar falhas no enrolamento do estator em motores de indução (Marques Cardoso et al., 1999). As componentes de corrente do vetor de Park são representas por $i_{d}$ (direta) e $i_{q}$ (quadratura). Essas componentes são obtidas pelas variáveis que representam as correntes das três fases do motor $\left(i_{a}, i_{b}\right.$ e $\left.i_{c}\right)$, isto é:

$$
\begin{gathered}
i_{d}=\sqrt{\frac{2}{3}} i_{a}-\frac{1}{\sqrt{6}} i_{b}-\frac{1}{\sqrt{6}} i_{c} \\
i_{q}=\frac{1}{\sqrt{2}} i_{b}-\frac{1}{\sqrt{2}} i_{c}
\end{gathered}
$$

Em condições de operação balanceada e saudável, a corrente do motor pela abordagem do vetor de Park é circular e centrada na origem do eixo de coordenadas. Sob condições de falta, este padrão é desviado do centro de acordo com a falta associada.

De forma geral, os métodos convencionais para monitorar as condições de uma máquina de indução utilizam das componentes espectrais da corrente do estator no regime permanente. Mesmo que as máquinas não operem sob as completas condições de regime permanente, técnicas como Park's Vector Approach (Marques Cardoso et al., 1999), o Extended Park's Vector Approach (EPVA) (Cruz and Cardoso, 2001; Barendse et al., 2009), o Support Vector Machine (SVM) (Radhika et al., 2010) e modelos dinâmicos (baseado no modelo transiente de quarta ordem) (Baccarini et al., 2010) são capazes de detectar curtos-circuitos nos enrolamentos do estator.

Outra técnica tradicional para detecção de falhas em motores é a Análise da Assinatura da Corrente do Motor (MCSA - Motor Current Signature Analysis) (Thomson and Fenger, 2001). O objetivo desta técnica é obter as componentes localizadas em $(1 \pm 2 s) f$ no espectro da corrente do estator para detectar faltas específicas (sendo $f$ a frequência das tensões de alimentação). No entanto, em Joksimovic and Penman (2000), mostra-se matematicamente e experimentalmente que componentes espectrais gerados por curtos em bobinas não são um indicador exato para definir falhas num enrolamento de estator.

O enrolamento do estator assimétrico e faltoso pode produzir harmônicas espaciais em qualquer onda no campo do entreferro. Entretanto, todas estas harmônicas variam numa única frequência, ou seja, a frequência da fonte de tensão senoidal da rede. As harmônicas do estator induzem correntes na gaiola do rotor e que refletem de volta ao estator, como novas harmônicas do campo do entreferro. Vista do estator, as harmônicas do entreferro, causadas pela corrente induzida do rotor, variam na frequência da seguinte forma:

$$
f_{r}=f_{1}\left[1 \pm \frac{\lambda n}{p}(1-s)\right]
$$

em que $f_{r}$ é o componente refletido no estator, $f_{1}$ é a frequência da fonte da rede elétrica, $p$ é o número de pares de pólos, $s$ é o escorregamento, $n$ é o número de barras do rotor e $\lambda$ é o número das harmônicas.

As harmônicas do campo do entreferro induzidas na corrente do estator apresentam-se na mesma frequência que uma harmônica produzida num motor saudável. Logo, uma falha no enrolamento do estator pode mudar a amplitude das harmônicas da corrente do estator, mas não produz nenhuma nova frequência no espectro da corrente. De acordo com esta análise, pode-se ser difícil detectar falhas do estator pelo espectro da corrente apenas pelo método MCSA (Joksimovic and Penman, 2000).

A Rede Neural Artificial (RNA) é uma das abordagens de Inteligência Artificial (IA) capaz de organizar as características dos sinais, assim como identificar e diagnosticar falhas presentes nos motores. Além disso, a não dependência de modelos matemáticos para sua efetiva implementação foi um dos fatores que as destacaram na resolução de problemas de falhas em MIT. Em Chow and Yee (1991) destaca-se uma das primeiras pesquisas na atribuição das RNAs para detecção de falhas incipientes em motores de indução do tipo gaiola de esquilo.

As falhas de curto-circuito no enrolamento do estator podem ser detectadas e classificadas por redes neurais das seguintes topologias: perceptron multicamada (MLP) (Ghate and Dudul, 2010; Tallam et al., 2003; Bouzid et al., 2008; Ghate and Dudul, 2009; Leite et al., 2009); RBF (Radial Base Function) (Wu and Chow, 2004; Ghate and Dudul, 2011); Hebbian (Martins et al., 2007); SOM (Self-Organizing Maps)(Ghate and Dudul, 2010); Elman neural network e Time Lagged Feed forward Network (TLFN) (Leite et al., 2009).

Em Ghate and Dudul (2010) define um classificador baseado nas redes neurais MLP e SOM, cujo objetivo é detectar curto-circuitos no enrolamento do estator, excentricidade dinâmica do rotor, ambas as falhas simultaneamente ou motor saudável. Os dados de entrada para as redes neurais foram obtidos a partir da corrente do estator. As três fases da corrente alternada do motor de indução são lidas e amostradas para o cálculo de parâmetros estatísticos, tais como o desvio padrão, va- 
lores de máximo e de mínimo do coeficiente de skewness e o coeficiente de kurtosis. A dimensionalidade destes parâmetros foi reduzida aplicando o método de Análise de Componentes Principais (PCA - Principal Component Analysis), o que gerou outras topologias de RNAs, pois a quantidade de neurônios foi reduzido tanto para a MLP (de 13 para 5 neurônios na camada escondida) quanto para a rede SOM (de 13 para 7 neurônios). Assim, foram testadas 4 topologias de RNAs (MLP, MLP reduzida, SOM e SOM reduzida) com diversas taxas de aprendizado e com várias funções de aprendizado. Em uma análise comparativa, a rede MLP reduzida atuou como um eficaz classificador de diagnóstico de faltas, obtendo-se $98.25 \%$ de acertos para o conjunto de dados destinados ao treinamento da rede e $96.22 \%$ para o conjunto de validação (dados não usados no treinamento).

Alternativamente, modelos compostos por redes neurais podem ser colocados em paralelo com os sistemas que devem ser monitorados, a fim de que os resultados obtidos pelas redes neurais sejam comparados com os dados reais de saída de um motor de indução (Su and Chong, 2007). Este procedimento foi aplicado a rede neural recorrente integrada à modelagem Bayesiana Dinâmica com o intuito de detectar enrolamentos curto-circuitados no estator, rachaduras nas esferas internas dos rolamentos ou um motor saudável (Cho et al., 2010). Para testar esta metodologia, três RNAs idênticas estruturalmente foram implementadas e testadas sob três motores com mesmas características, mas cada uma com um dos objetivos citados. Conclui-se que o algoritmo proposto identificou os motores que estavam operando anormalmente e que a falha no rolamento foi mais fácil de ser detectada do que a falha no enrolamento.

Qualquer tipo de falha no enrolamento do estator pode levar à parada do motor, o que seria inaceitável em algumas aplicações. A adição de mais fases num motor de indução garante a confiabilidade e a operação contínua do sistema, caso uma das fases de um motor trifásico falhe (Kianinezhad et al., 2008). Adicionalmente, algoritmos de controle formados pela lógica fuzzy e pelo slidingmode foram também usados para obter, com alta precisão, o funcionamento de uma máquina de indução de seis fases em modo saudável ou não (Fnaiech et al., 2010).

Dentre as metodologias da inteligência artificial, a lógica fuzzy é bem utilizada em sistemas de monitoramento de falhas no enrolamento do estator (Rodriguez and Arkkio, 2008; Zidani et al., 2003; Mini et al., 2010; Kumar et al., 2010; Karakose et al., 2010). A lógica fuzzy surgiu como um meio de representar a imprecisão inerente ao processo por meio de termos lingüísticos, assim como uma maneira de expressar o conhecimento humano por uma forma mais natural (Zadeh, 1965). As variáveis que representam a situação do motor serão transformadas em conjuntos fuzzy para que, posteriormente, sejam analisados pelo conjunto de regras do sistema fuzzy, o qual foi formulado por especialistas naquele processo. O conhecimento armazenado por estes sistemas toma decisões que tem gerado resultados com alta percentagem de acertos. Devido a isto, os sistemas de controle avançados estão, progressivamente, empregando os conceitos da teoria fuzzy desde os anos 80 (Dos Santos Coelho et al., 2003). Os controladores fuzzy são capazes de analisar parâmetros não-lineares, variantes no tempo e com informações imprecisas, além de apresentarem um desempenho igual ou superior aos controladores convencionais (Callai et al., 2007).

A lógica fuzzy, em Mini et al. (2010), é aplicada para diagnosticar as condições do estator de um motor de indução e as condições de um circuito aberto numa das fases, a partir da amplitude da corrente do estator. As variáveis de entrada são as três fases da corrente $\left(I_{a}, I_{b}, I_{c}\right)$, interpretadas como variáveis linguísticas $T(Q)=\{$ zero, pequeno, médio, grande $\}$, sendo $Q=I_{a}, I_{b}, I_{c}$. A variável de saída é o termo $C M$, que significa condições do motor, a qual foi também definida como variável linguística $T(C M)=\{$ bom, danificado, seriamente danificado, circuito aberto_ $A$, circuito aberto_B, circuito aberto_ $C\}$. O sistema de decisão fuzzy obteve exatidão em seu diagnóstico.

Em Karakose et al. (2010), o autor apresenta choquet fuzzy integral para diagnosticar falhas no estator e quebras na barra do rotor frente às duas fases de processamento que dividiu o sistema. Na primeira fase, características são extraídas do sinal de corrente do motor para estimar a gravidade da falta. Na segunda fase, diferentes algoritmos de diagnóstico (rede MLP, SVM, AG, AIS - Artificial Immune System, e FAIS - Fuzzy Artificial Immune System) são combinados ao choquet fuzzy integral para diagnosticar com exatidão as falhas do motor, sendo o SVM o algoritmo de diagnóstico que obteve melhores resultados dentre os demais.

Outra metodologia para diagnosticar falhas no estator e quebras na barra do rotor está descrito em Akin et al. (2011). Neste artigo, três sistemas foram implementados em paralelo num FPGA Field Programmable Gate Array, cujo tempo de resposta e funcionalidade caracterizou todo o conjunto como um sistema online em tempo real. O primeiro sistema é uma RNA com o objetivo de estimar alguma anomalia no motor, a partir da leitura de uma das fases da corrente. O segundo é a implementação da LF para diagnosticar falhas no estator, extraindo-se informações 
da amplitude das três fases da corrente do motor. Por fim, o terceiro sistema detecta falhas na barra do rotor usando o algoritmo de seleção negativa por dados obtidos em uma fase da corrente. Os resultados foram satisfatórios, os quais são obtidos, sobretudo, num tempo de processamento de dois milissegundos.

Com objetivo de produzir metodologias mais eficazes, duas ou mais técnicas da inteligência artificial podem ser combinadas e implementadas num único sistema computacional, sendo intitulado por sistema híbrido avançado ou inteligente híbrido. Os sistemas híbridos avançados podem ser classificados de acordo com sua constituição, isto é, seqüencial, auxiliar ou incorporado. No sistema híbrido seqüencial, executa-se, primeiramente, a técnica de um subsistema e em seguida a técnica do outro. O sistema híbrido auxiliar também é formado por dois subsistemas, sendo que um deles tem a função de realizar uma tarefa auxiliar ao subsistema principal. Em D'Angelo et al. (2011), falhas nos enrolamentos do estator foram analisadas por um sistema híbrido auxiliar mediante análise dos sinais da corrente do estator e da velocidade angular do rotor. Neste artigo, inicialmente, os dados foram processados pela clusterização fuzzy, cujos centros dos clusters foram determinados por uma rede neural do tipo Kohonen, seguido da execução do algoritmo Metropolis-Hastings.

Por outro lado, os sistemas neuro-fuzzy são geralmente técnicas ideais para definir um sistema híbrido incorporado, ou seja, não há como separar os procedimentos de cada subsistema. No sistema neuro-fuzzy a inferência fuzzy é implementada segundo a estrutura de uma rede neural, o que fornece o raciocínio heurístico do processo de detecção de falhas. Além disso, as suas aplicações são capazes de descrever as condições reais da falha do estator, tais como aquelas abordagens formuladas em Goode (1995a), Goode (1995b), Caminhas et al. (1996), Ballal et al. (2007) e Tran et al. (2009).

\section{FALHAS NO ROTOR}

Segundo Vas (1993), as falhas oriundas do rotor, cuja estrutura é do tipo gaiola de esquilo, podem ser identificadas nos componentes de sua estrutura principal (corpo do rotor). Assim, a Tabela 2 descreve as respectivas falhas dos componentes.

Dentre as falhas classificadas, a quebra na barra do rotor e as rachaduras nos anéis finais compartilham de $5 \%$ a $10 \%$ das falhas em máquinas de indução (Bellini et al., 2008a). Para estas falhas, a abordagem do vetor de Park (Karami et al., 2010a; Pezzani et al., 2010) e, principalmente, a MCSA foram destacadas na litera-
Tabela 2: Falhas dos Componentes do Rotor

\begin{tabular}{|c|c|c|}
\hline Rotor & Componentes & Tipos de Falhas \\
\hline \multirow{4}{*}{ Barra } & Eixo & Estrutura física \\
\hline & \multirow{2}{*}{ Corpo } & Ponto quente no núcleo \\
\hline & & Integridade do corpo \\
\hline & Anéis & $\begin{array}{l}\text { Rachadura ou quebra dos } \\
\text { anéis }\end{array}$ \\
\hline
\end{tabular}

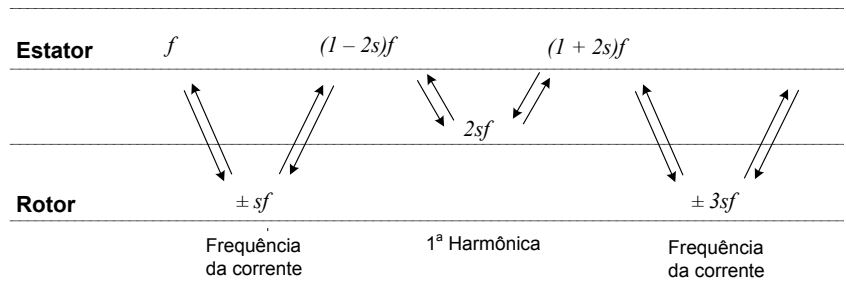

Figura 1: Efeitos nas correntes do estator e do rotor devido à da assimetria do rotor.

tura, conforme as técnicas apresentadas em Ayhan et al. (2008), Concari et al. (2008), Puche-Panadero et al. (2009), Riera-Guasp et al. (2010) e Concari et al. (2010).

Um motor de indução operando com uma falha no rotor gera uma seqüência negativa de corrente devido à assimetria do rotor. Isto induz uma componente principal na corrente do estator, a qual varia na frequência de $(1-2 s) f$. Além disso, as frequências de assimetria do rotor são $\pm s f, \pm 3 s f, \ldots$; como esboçado na Fig. 1 (Bellini et al., 2008a).

A dificuldade em se detectar falhas nos rotores está justamente na classificação das linhas de frequência presentes nos sinais. Para minimizar este problema e obter uma ferramenta eficiente de detecção de falhas, os sistemas inteligentes têm sido também utilizados para mapear estes processos considerados complexos.

As redes neurais combinadas com diferentes métodos compõem diversas estruturas para um sistema de diagnóstico, principalmente naqueles métodos responsáveis pela extração de características do sinal. Como exemplo, a estrutura formada pela Current Signal Analysis (CSA) e a rede neural MLP podem analisar seis diferentes situações do rotor: de uma a três quebras na barra do rotor, barra com alta resistência, quebra nos anéis finais ou rotor saudável, conforme constatado em Arabaci and Bilgin (2009). Neste artigo, a rede neural foi usada tanto para detectar como para classificar as falhas do rotor, obtendo-se $100 \%$ e $98,33 \%$ de exatidão, respectivamente. 
Já em Su and Chong (2007), uma rede neural MLP combinada com a transformada de Fourier (STFT) utilizam do espectro de sinais de vibração de motores saudáveis a fim de treinar a rede. Uma vez que o modelo é estabelecido, sinais de vibração obtidos sob condições de falhas serão aplicados ao sistema para gerar uma análise residual e, posteriormente, calcular o nível da falha. O indicador do nível da falha está baseado na distorção entre o sinal de vibração em condições saudáveis e o resultado do residual na presença de falhas. Neste estudo, as falhas analisadas foram quatro situações diferentes de barras quebradas e excentricidade. Para todas as falhas, os resíduos de vibração resultantes sobressaiam à linha de base que limitava a condição saudável para um motor, além de que a magnitude do residual aumentava nitidamente com a severidade das falhas.

Outro exemplo é o sistema formado pela transformada Wavelet Packet Decomposition (WPD) e a rede MLP, que pode identificar falhas nas barras do rotor através das frequências do escorregamento e da corrente do estator (Sadeghian et al., 2009). Este sistema foi dividido em quatro passos: aquisição de dados, préprocessamento, detecção de falta e pós-processamento. No pré-processamento, o sinal da corrente do estator é transformado para o domínio tempo-frequência pela WPD e que, conseqüentemente, gera os coeficientes característicos. Esses coeficientes possuem resoluções de diferentes frequências, e junto com a frequência de escorregamento treinam a rede neural. A etapa do pósprocessamento armazena os diferentes tipos de faltas encontrados pela rede num banco de dados para, posteriormente, ser usado no aperfeiçoamento do algoritmo de detecção de falhas.

Outras arquiteturas e topologias de redes neurais também foram desenvolvidas para falhas no rotor, principalmente para quebras nas barras do rotor, tais como: MLP (Ayhan et al., 2006; Pawlak and Kowalski, 2010; Bouzid et al., 2010), SOM (Cupertino et al., 2005), General Regression Neural Networks (GRNN)(Kaminski et al., 2010), Kohonen (Vas, 1999).

Além das redes neurais, a lógica fuzzy tem também atuado tanto na deteç̧ão de falha (Zouzou et al., 2009) quanto na exatidão e quantificação do índice de falha dos rotores (Karakose et al., 2010; Razik et al., 2009). Em Razik et al. (2009) é descrita uma metodologia constituída pela transformada Concordia, Algoritmo Genético (AG) e um sistema baseado na lógica fuzzy. O primeiro é responsável pela filtragem e síntese das três linhas da corrente do estator; o AG busca a falha no espectro de frequência; e o nível de gravidade da falha é definido pela abordagem fuzzy. Este sistema foi capaz de infor- mar ao operador da máquina, por meio de indicadores luminosos, sobre o estado da mesma, o qual poderia ser classificado como saudável, falha incipiente no rotor, ou presença de uma barra quebrada no rotor. Tais estados foram notificados e testados ao operar o motor sob $50 \%$, $75 \%$ ou $100 \%$ de sua carga nominal.

A LF aliada à entropia da informação compôs um sistema em tempo-real de detecção de falhas simples ou compostas, sendo implementado em hardware num dispositivo FPGA (Romero-Troncoso et al., 2011). A análise da entropia da informação ofereceu referências quantitativas da informação inserida numa fase da corrente, e a inferência da LF, aplicada sob cada valor retornado da entropia, fez uma exata identificação da condição da falha simples ou múltipla, que poderia ser quebra na barra do rotor, desbalanceamento ou falhas nos rolamentos. O sistema gerou $100 \%$ de exatidão para motores saudáveis e com as três falhas juntas. Porém, a identificação das outras combinações de falhas (individuais e múltiplas) diminui a eficiência, mas os resultados ainda foram considerados satisfatórios.

Destaca-se também a presença de sistemas neuro-fuzzy na classificação de falhas que, combinados à outras metodologias, são responsáveis pela seleção e extração de características do conjunto de dados. Dentre estas metodologias, destacam-se a árvore de classificação e regressão (CART) (Tran et al., 2009) e o analisador threshold representado por uma curva empírica da velocidadetorque (Tan and Huo, 2005). Além disso, destaca-se também a formulação de um controlador neuro-fuzzy para tolerar efeitos e reações do motor na presença de barras quebradas no rotor, conforme investigado em Uddin et al. (2010).

Em relação à localização das frequências que representam falhas no rotor, em Razik et al. (2010) é utilizado o algoritmo Particle Swarm Otimization (PSO) (Kennedy and Eberhart, 1995) para explorar o espaço de busca e extrair os espectros de frequência. O algoritmo PSO é uma técnica de otimização que simula a habilidade de um indivíduo dentro de uma sociedade durante um processo de conhecimento. Em outras palavras, é um algoritmo que modela a exploração do espaço do problema por uma população de indivíduos, tal que suas buscas são influenciadas por bons resultados adquiridos pelos indivíduos da população e por ele próprio (Kennedy, 1997).

Sob uma carga nominal de $75 \%$, o algoritmo PSO em Razik et al. (2010) identificou frequências na linha da corrente do estator, que representavam uma barra quebrada no rotor, estando o escorregamento já no regime 

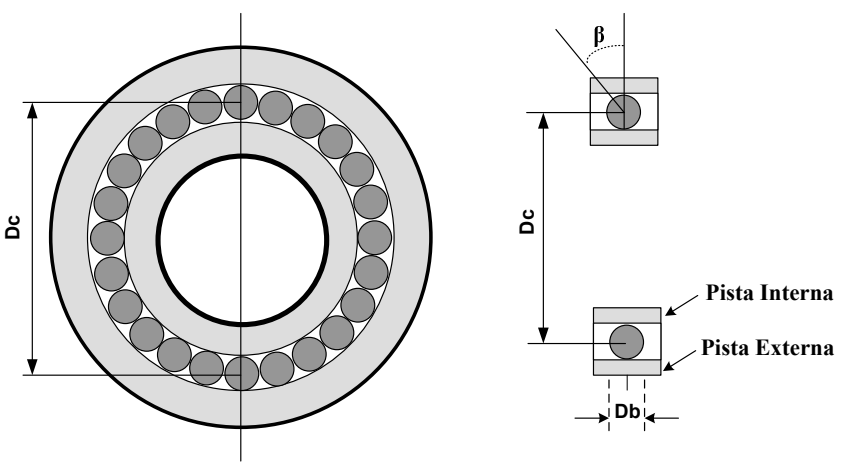

Figura 2: Estrutura e parâmetros de um rolamento.

permanente, em aproximadamente 100 iterações. Durante os testes, observou-se que as partículas que não apresentavam bom desempenho desapareciam durante as iterações. Além disso, deduziu-se o bom desempenho do algoritmo PSO no caso em que o motor estivesse operando sob carga leve, pois nessa situação as linhas de falhas estariam muito próximas uma das outras e poderiam confundir o processo de otimização. No entanto, o algoritmo PSO evita soluções locais independentemente do tamanho do espaço de busca.

Recentemente, uma metodologia inspirada no sistema imunológico humano tem se tornado destaque na área da inteligência computacional, denominada de sistema imunológico artificial (Castro and Timmis, 2002; Laurentys et al., 2010). Baseado nas teorias deste sistema, um novo método de programação, chamado Programação de Seleção de Clones (CSP), foi proposto em Gan et al. (2009a) para melhorar a eficácia da codificação de programas e dos mecanismos de busca. Já em Gan et al. (2009b), o CSP foi aplicado no classificador responsável pela detecção de falhas da máquina a partir dos sinais de vibração.

\section{FALHAS NOS ROLAMENTOS}

Um rolamento consiste de anéis interno e externo, entre os quais um conjunto de esferas ou rolos gira, formandose então uma pista entre os anéis, conforme ilustrado na Fig. 2. Falhas de rolamento podem ser oriundas dos anéis internos, dos anéis externos ou dos elementos girantes. Tais falhas se desenvolvem devido à vibração, stress interno, desalinhamento do eixo, excentricidade, corrente no rolamento, poeira ou corrosão.

Em Vas (1993), as falhas do rolamento foram identificadas ao associar a frequência de vibração do eixo com características dos elementos do rolamento, as quais podem ser descritas pelas seguintes equações:
- defeitos na pista externa:

$$
f_{o}=\left(\frac{N}{2}\right) f_{r}\left[1-\left(\frac{D_{b}}{D_{c}}\right) \cos \beta\right]
$$

- defeitos na pista interna:

$$
f_{i}=\left(\frac{N}{2}\right) f_{r}\left[1+\left(\frac{D_{b}}{D_{c}}\right) \cos \beta\right]
$$

- defeitos nos elementos rolantes:

$$
f_{b}=\left(\frac{D_{c}}{D_{b}}\right) f_{r}\left\{1-\left[\left(\frac{D_{b}}{D_{c}}\right) \cos \beta\right]^{2}\right\}
$$

- defeitos na caixa de rolamento:

$$
f_{t}=\left(\frac{f_{r}}{2}\right)\left[1-\left(\frac{D_{b}}{D_{c}}\right) \cos \beta\right]
$$

sendo que $N$ é o número de elementos rolantes ou esferas; $f_{r}$ é a frequência rotacional do rotor; $D_{b}$ é o diâmetro da esfera; $D_{c}$ é o diâmetro do campo da esfera; e $\beta$ é o ângulo de contato da esfera com a pista.

Vários trabalhos aplicaram redes neurais em sistemas de diagnóstico de falhas nos rolamentos, como em $\mathrm{Wu}$ and Chow (2004), onde é apresentado uma rede neural $\mathrm{RBF}$, com auto-ajuste no número de neurônios na camada escondida, segundo a metodologia da rede SOM. O espectro da corrente e os sinais de vibração do motor forneceram os dados de entrada para as redes neurais. Em relação às falhas, foram induzidos níveis diferentes de desbalanceamento elétrico na alimentação do motor e três extensões de relaxamento no parafuso responsável pela fixação do motor na bancada, provocando-se, conseqüentemente, vibrações de natureza elétrica e mecânica no núcleo do rotor. Os resultados mostraram $100 \%$ de acerto na identificação de falhas mecânicas durante os testes da rede, enquanto que as falhas elétricas obtiveram $85.7 \%$, ao passo que o motor sob condições normais obteve $96.0 \%$.

A rede neural do tipo MLP também foi implementada para identificar falhas em rolamentos, conforme formulações descritas em Kankar et al. (2011), Li et al. (2000), Samanta and Nataraj (2009), Yang (2010), Castejón et al. (2010) e Mahamad and Hiyama (2010). Entretanto, em alguns sistemas, a rede MLP aparece integrada a diferentes técnicas, como o SVM (Kankar et al., 2011) e o algoritmo PSO (Samanta and Nataraj, 2009). Nestes sistemas, um pré-processamento dos sinais se fez necessário para compilar as entradas da rede neural, tais como aqueles baseados em métodos estatísticos (Kankar et al., 2011), analisador biespectral baseado em Hilbert (Yang, 2010) e Multiresolution analysis 
(MRA) (Castejón et al., 2010). Além da rede neural MLP, comparou-se também em Mahamad and Hiyama (2010) os resultados gerados pelas redes neurais de tipo Elman, RBF e pelo sistema neuro-fuzzy (ANFIS).

Em Mahamad and Hiyama (2008), o uso único da rede de Elman foi capaz para identificar falhas nos rolamentos por meio dos sinais de vibração. Em Yan et al. (2010), espectros de energia extraídos pela transformada Wavelet Packet foram repassados ao algoritmo PCA, que selecionou características e encaminhou ao algoritmo da lógica fuzzy, responsável em unir dados que representam defeitos iniciantes nas esferas, nas pistas interna e externa do rolamento, com diâmetros de 7, 14 e 21 milímetros. A metodologia proposta foi eficiente para diagnosticar as falhas incipientes; porém resultados mais promissores foram alcançados ao aumentar a estratificação dos padrões de falhas.

Para aquelas aplicações usando sistemas híbridos, observou-se aqui uma diversificação de variáveis atuando como dados de entrada em sistemas de tipo neuro-fuzzy para monitoramento de falhas em rolamentos. Medidas de velocidade, torque e corrente foram os dados de entrada gerados pelo motor para os sistemas ANFIS e FALCON (Fuzzy Adaptive Learning Control/Decision Network) formulado em Altug and Trussell (1999); ruído, temperatura do mancal, temperatura do enrolamento, velocidade e corrente foram os dados de entrada para aquele sistema ANFIS apresentado em Ballal et al. (2007); as três fases da corrente do estator, a velocidade e o torque da carga foram as variáveis de entrada para o sistema neuro-fuzzy descrito em Abu-Rub et al. (2010); e apenas os sinais de vibração foram usados por sistemas ANFIS desenvolvidos em Mahamad and Hiyama (2010) e Zhang et al. (2010), e IFAM (improved Fuzzy ARTMAP) em Xu et al. (2009).

\section{FALHAS DE EXCENTRICIDADE}

Excentricidade num motor é uma folga desigual existente entre o estator e o rotor (Vas, 1993), e que é classificada como estática, dinâmica ou mista. Na excentricidade estática, o centro de rotação do eixo é deslocado do centro original a uma distância que permanece fixa. Por outro lado, na excentricidade dinâmica, o centro de rotação permanece na posição original, mas o eixo é deslocado. Logo, a excentricidade mista representa o centro e o eixo de rotação deslocados de suas respectivas posições de origem.

A excentricidade de folga é uma falha comum nos motores de indução trifásico. Porém, devido às algumas imperfeições de fabricação e de desempenho, até $10 \%$ de ex- centricidade é permitido (Awadallah and Morcos, 2003). Uma excentricidade pode ser causada por vários fatores, tais como mau posicionamento do mancal durante a montagem do motor, desgaste do rolamento, inclinação do eixo do rotor (Ong et al., 2000). A excentricidade gera uma força no rotor que tenta arrancá-lo dos mancais suportados pelo estator, causando um significante aumento em seus desgastes.

Dentre as técnicas convencionais, MCSA foi usada extensivamente para diagnosticar a excentricidade. As frequências específicas relacionadas à esta falta são dadas por (Vas, 1993):

$$
f_{e c}=\left[\left(k R \pm n_{d}\right) \frac{(1-s)}{p} \pm v\right] f_{1}
$$

onde $k$ é um inteiro positivo, $R$ é o número das barras do rotor, $p$ é o número da pares de pólos, $n_{d}$ é a ordem da excentricidade, $s$ é o escorregamento do motor, $v$ é a ordem das harmônicas, e $f_{1}$ é a frequência de alimentação.

Sinais de vibração, tensão e corrente do estator são monitorados para detectar falhas relacionadas à excentricidade. Na literatura, estes sinais foram também analisados por sistemas inteligentes, tais como a rede MLP (Ghate and Dudul, 2009; Su and Chong, 2007; Huang et al., 2007) e a rede RBF aliada à rede MLP (RBFMLP) (Ghate and Dudul, 2011).

Em Ghate and Dudul (2011), alguns parâmetros estatísticos foram calculados a partir da corrente do estator, os quais foram selecionados pela técnica do PCA, e encaminhados à rede RBF-MLP. Ou seja, a primeira camada neural dessa rede assemelha-se à primeira camada de uma rede RBF, que contém a função Gausiana como função de aprendizado, composta por regras competitivas e métricas adequadas para se obter convergência para um mínimo local. Da segunda até a última camada é a representação da rede MLP, que foi anexada à camada da RBF com a função de otimizar a topologia da rede proposta. A rede neural em cascata RBF-MLP notificou satisfatoriamente as falhas excentricidade no rotor e curto-circuito entre os enrolamentos do estator.

\section{PROBLEMAS EM ABERTO E NOVAS TENDÊNCIAS}

Dentre os artigos citados, observaram-se algumas ressalvas nos sistemas de diagnóstico de falhas que não obtiveram soluções eficientes. A identificação do número de voltas curto-circuitadas numa fase do estator, e a notificação de falhas provindas fora do MIT, como a tensão ou corrente desbalanceada, são exemplos destes proble- 
Tabela 3: Resumo dos Artigos que Utilizam Sistemas Inteligentes na Identificação de Falhas em MIT.

\begin{tabular}{|c|c|c|}
\hline Tipos de Falhas & Classificador & Referências \\
\hline \multirow{4}{*}{ Enrolamento do estator } & RNA & $\begin{array}{l}\text { Tallam et al. (2003), Wu and Chow (2004), Martins et al. (2007), Su } \\
\text { and Chong (2007), Bouzid et al. (2008), Ghate and Dudul (2009), } \\
\text { Leite et al. (2009), Ghate and Dudul (2010), Cho et al. (2010), Ghate } \\
\text { and Dudul (2011) }\end{array}$ \\
\hline & LF & $\begin{array}{l}\text { Zidani et al. (2003), Rodriguez and Arkkio (2008), Fnaiech et al. } \\
\text { (2010), Mini et al. (2010), Kumar et al. (2010), Karakose et al. (2010), } \\
\text { Mini et al. (2010), Akin et al. (2011) }\end{array}$ \\
\hline & RNA e LF & D'Angelo et al. (2011) \\
\hline & Neuro-fuzzy & $\begin{array}{l}\text { Goode (1995a), Goode (1995b), Caminhas et al. (1996), Ballal et al. } \\
\text { (2007), Tran et al. (2009) }\end{array}$ \\
\hline \multirow{5}{*}{ Barras do rotor } & RNA & $\begin{array}{l}\text { Cupertino et al. (2005), Ayhan et al. (2006), Su and Chong (2007), } \\
\text { Arabaci and Bilgin (2009), Sadeghian et al. (2009), Kaminski et al. } \\
\text { (2010), Pawlak and Kowalski (2010), Bouzid et al. (2010) }\end{array}$ \\
\hline & $\mathrm{LF}$ & $\begin{array}{l}\text { Zouzou et al. (2009), Karakose et al. (2010), Razik et al. (2009), } \\
\text { Romero-Troncoso et al. (2011) }\end{array}$ \\
\hline & Neuro-fuzzy & Tan and Huo (2005), Tran et al. (2009), Uddin et al. (2010) \\
\hline & PSO & Razik et al. (2010) \\
\hline & CSP & Gan et al. (2009b) \\
\hline \multirow[t]{3}{*}{ Rolamento } & RNA & $\begin{array}{l}\text { Li et al. (2000), Wu and Chow (2004), Mahamad and Hiyama (2008), } \\
\text { Samanta and Nataraj (2009), Onel et al. (2009), Yang (2010), Cas- } \\
\text { tejón et al. (2010), Mahamad and Hiyama (2010), Cho et al. (2010), } \\
\text { Kankar et al. (2011) }\end{array}$ \\
\hline & LF & Yan et al. (2010), Romero-Troncoso et al. (2011) \\
\hline & Neuro-fuzzy & $\begin{array}{l}\text { Ballal et al. (2007), Xu et al. (2009), Abu-Rub et al. (2010), Mahamad } \\
\text { and Hiyama (2010), Zhang et al. (2010) }\end{array}$ \\
\hline Excentricidade & RNA & $\begin{array}{l}\text { Su and Chong (2007), Huang et al. (2007), Ghate and Dudul (2009), } \\
\text { Ghate and Dudul (2011) }\end{array}$ \\
\hline
\end{tabular}

mas. O reconhecimento da tensão desbalanceada induz a falsos alarmes, o que prejudica o desempenho do sistema de diagnóstico (Leite et al., 2009). Igualmente, há dificuldades nos sistemas de diagnóstico de falhas que já foram solucionadas, mas que ainda podem ser mais bem explorados. Neste caso, apontam-se as técnicas computacionais que foram aplicadas aos parâmetros estatísticos dos MIT com o intuito de filtrá-los para os sistemas inteligentes. Exemplos destes métodos são: PCA (Ghate and Dudul, 2010), CART (Tran et al., 2009) ou algoritmos de otimização.

Por outro lado, novas tendências teóricas e tecnológicas têm sido apontadas e encorajadas pelos artigos investigados, tais como as seguintes:

- O uso de inversores para notificar falhas de origem interna e externa ao MIT.

- Sistema de identificação e classificação de falhas elétricas e mecânicas baseado em métodos compu- tacionais, como o CSP, Choquet Fuzzy Integral e GRNN.

- Combinação de diferentes abordagens com aquelas advindas dos sistemas inteligentes, tais como: CART e ANFIS, MLP e SVM, MLP e PSO, AG e LF, AG e PSO.

\section{CONCLUSÃO}

Este artigo apresentou uma visão geral das aplicações mais relevantes dos sistemas inteligentes na identificação e diagnóstico de falhas de motores de indução trifásico. Destacou-se a presença de diferentes topologias de RNAs, lógica fuzzy, AG, PSO, CSP, e sistemas neurofuzzy que representam os sistemas híbridos avançados. Portanto, a Tabela 3 cita os principais estudos que foram referenciados neste texto, destacando-se as falhas e os respectivos sistemas inteligentes utilizados na sua identificação. 
Pela Tabela 3, observa-se a expressiva quantidade de trabalhos que utilizaram as RNAs para identificar as falhas dos MIT. Isto se deve às diferentes topologias de redes, à baixa complexidade computacional em sua implementação, à capacidade de organizar os dados, além de outras particularidades que cada autor destacou em seus trabalhos. Dentre os artigos citados, alguns desenvolveram sistemas inteligentes que abordaram múltiplas e simultâneas falhas no motor, e outros que destacaram a presença de inversores no acionamento dos MIT.

Conclui-se que as metodologias dos sistemas inteligentes se destacam por várias particularidades, dentre as quais a possibilidade de treinamento a partir dos sinais do motor em ambos os domínios (tempo ou frequência), predição de falhas incipientes devido às anomalias de operação, reconhecimento de padrões e mapeamento não-linear no monitoramento das condições do motor, emulação e implementação do conhecimento humano, e classificação das falhas.

O expressivo número de trabalhos com resultados bem promissores prova a eficiência destes sistemas inteligentes na automatização dos processos de diagnóstico de falhas, os quais podem aprimorar ainda mais os sistemas a serem desenvolvidos.

\section{AGRADECIMENTOS}

Os autores agradecem ao CNPq (Processo 143635/20085) pelos auxílios financeiros concedidos.

\section{REFERÊNCIAS}

Abu-Rub, H., Moin Ahmed, S., Iqbal, A., Rahimian, M. and Toliyat, H. (2010). Incipient bearing fault diagnostics for inverter fed induction motor drive using ANFIS, Proc. The XIX International Conference on Electrical Machines (ICEM 2010), pp. 1-5.

Akin, E., Aydin, I. and Karakose, M. (2011). FPGA based intelligent condition monitoring of induction motors: detection, diagnosis, and prognosis, Proc. IEEE International Conference on Industrial Technology (ICIT), pp. 373-378.

Altug, S. and Trussell, H. (1999). Fuzzy inference systems implemented on neural architectures for motor fault detection and diagnosis, IEEE Transactions on Industrial Electronics 46(6): 1069-1079.

Arabaci, H. and Bilgin, O. (2009). Automatic detection and classification of rotor cage faults in squirrel cage induction motor, Neural Computing and Applications 19(5): 713-723.
Awadallah, M. A. and Morcos, M. M. (2003). Application of AI tools in fault diagnosis of electrical machines and drives-an overview, IEEE Transactions on Energy Conversion 18(2): 245-251.

Ayhan, B., Chow, M.-Y. and Song, M.-H. (2006). Multiple discriminant analysis and neural-networkbased monolith and partition fault-detection schemes for broken rotor bar in induction motors, IEEE Transactions on Industrial Electronics 53(4): 12981308.

Ayhan, B., Trussell, H. J., Chow, M.-Y. and Song, M.H. (2008). On the use of a lower sampling rate for broken rotor bar detection with DTFT and ARbased spectrum methods, IEEE Transactions on Industrial Electronics 55: 1421-1434.

Baccarini, L. M. R., Menezes, B. R. and Caminhas, W. M. (2010). Fault induction dynamic model, suitable for computer simulation: Simulation results and experimental validation, Mechanical Systems and Signal Processing 24(1): 300-311.

Baccarini, L. M. R., Rocha e Silva, V. V., De Menezes, B. R. and Caminhas, W. M. (2011). SVM practical industrial application for mechanical faults diagnostic, Expert Systems with Applications 38(6): 6980-6984.

Ballal, M. S., Khan, Z. J., Suryawanshi, H. M. and Sonolikar, R. L. (2007). Adaptive neural fuzzy inference system for the detection of inter-turn insulation and bearing wear faults in induction motor, IEEE Transactions on Industrial Electronics 54(1): 250-258.

Barendse, P. S., Herndler, B., Khan, M. A. and Pillay, P. (2009). The application of wavelets for the detection of inter-turn faults in induction machines, Proc. IEEE International Electric Machines and Drives Conference, pp. 1401-1407.

Bellini, A., Filippetti, F., Franceschini, G. and Tassoni, C. (2000). Closed-loop control impact on the diagnosis of induction motors faults, IEEE Transactions on Industry Applications 36(5): 1318-1329.

Bellini, A., Filippetti, F., Tassoni, C. and Capolino, G. A. (2008a). Advances in diagnostic techniques for induction machines, IEEE Transactions on Industrial Electronics 55(12): 4109-4126.

Bellini, A., Immovilli, F., Rubini, R. and Tassoni, C. (2008b). Diagnosis of bearing faults of induction machines by vibration or current signals: a critical comparison, Proc. IEEE Industry Applications Society Annual Meeting, pp. 1-8. 
Bonnett, A. and Yung, C. (2008). Increased efficiency versus increased reliability, IEEE Industry Applications Magazine 14(1): 29-36.

Bouzid, M. B. K., Champenois, G., Bellaaj, N. M., Signac, L. and Jelassi, K. (2008). An effective neural approach for the automatic location of stator interturn faults in induction motor, IEEE Transactions on Industrial Electronics 55(12): 4277-4289.

Bouzid, M., Champenois, G., Bellaaj, N. and Jelassi, K. (2010). Automatic and robust diagnosis of broken rotor bars fault in induction motor, Proc. The XIX International Conference on Electrical Machines (ICEM 2010), Vol. 2, pp. 1-7.

Briz, F., Degner, M. W., Garcia, P. and Diez, A. B. (2008). High-frequency carrier-signal voltage selection for stator winding fault diagnosis in inverterfed AC machines, IEEE Transactions on Industrial Electronics 55(12): 4181-4190.

Callai, T. C., Dos Santos Coelho, L. and Coelho, A. A. R. (2007). Controle nebuloso adaptativo por modelo de referência: Projeto e aplicação em sistemas não-lineares, Controle \&\} Automação 18(4): 479489.

Caminhas, W. M., Tavares, H. and Gomide, F. (1996). A neurofuzzy approach for fault diagnosis in dynamic systems, Proc. IEEE International Conference on Fuzzy Systems, pp. 2032-2037.

Castejón, C., Lara, O. and García-Prada, J. C. (2010). Automated diagnosis of rolling bearings using MRA and neural networks, Mechanical Systems and Signal Processing 24(1): 289-299.

Castro, L. N. D. and Timmis, J. (2002). Artificial Immune Systems: A New Computational Intelligence Approach., 1st edn, Springer, London.

Cheng, S., Zhang, P. and Habetler, T. G. (2011). An impedance identification approach to sensitive detection and location of stator turn-to-turn faults in a closed-loop multiple-motor drive, IEEE Transactions on Industrial Electronics 58(5): 1545-1554.

Cho, H. C., Knowles, J., Fadali, M. S. and Lee, K. S. (2010). Fault detection and isolation of induction motors using recurrent neural networks and dynamic bayesian modeling, IEEE Transactions on Control Systems Technology 18(2): 430-437.

Chow, M. and Yee, S. (1991). Methodology for on-line incipient fault detection in single-phase squirrel-cage induction motors using artificial neural networks, IEEE Transactions on Energy Conversion 6(3): 536-545.

Concari, C., Franceschini, G. and Tassoni, C. (2008). Differential diagnosis based on multivariable monitoring to assess induction machine rotor conditions, IEEE Transactions on Industrial Electronics 55(12): 4156-4166.

Concari, C., Franceschini, G. and Tassoni, C. (2010). A MCSA procedure to diagnose low frequency mechanical unbalances in induction machines, Proc. The XIX International Conference on Electrical Machines (ICEM 2010), pp. 1-6.

Cruz, S. M. A. and Cardoso, A. J. M. (2001). Stator winding fault diagnosis in three-phase synchronous and asynchronous motors, by the extended Park's vector approach, IEEE Transactions on Industry Applications 37(5): 1227-1233.

Cupertino, F., Giordano, V., Mininno, E. and Salvatore, L. (2005). Application of supervised and unsupervised neural networks for broken rotor bar detection in induction motors, Proc. IEEE International Conference on Electric Machines and Drives, pp. 1895-1901.

D'Angelo, M. F. S. V., Palhares, R. M., Takahashi, R. H. C., Loschi, R. H., Baccarini, L. M. R. and Caminhas, W. M. (2011). Incipient fault detection in induction machine stator-winding using a fuzzyBayesian change point detection approach, Applied Soft Computing 11(1): 179-192.

Dos Santos Coelho, L., Da M. Almeida, O. and Coelho, A. A. R. (2003). Projeto e estudo de caso da implementação de um sistema de controle nebuloso, Controle $\mathcal{E}$ Automação 14(1): 20-29.

Filippetti, F., Franceschini, G., Tassoni, C. and Vas, P. (2000). Recent developments of induction motor drives fault diagnosis using AI techniques, IEEE Transactions on Industrial Electronics 47(5): 994 1004.

Fnaiech, M. A., Betin, F., Capolino, G.-A. and Fnaiech, F. (2010). Fuzzy logic and sliding-mode controls applied to six-phase induction machine with open phases, IEEE Transactions on Industrial Electronics 57(1): 354-364.

Frosini, L. and Bassi, E. (2010). Stator current and motor efficiency as indicators for different types of bearing faults in induction motors, IEEE Transactions on Industrial Electronics 57(1): 244-251. 
Gan, Z., Chow, T. and Chau, W. (2009a). Clone selection programming and its application to symbolic regression, Expert Systems with Applications 36(2): 3996-4005.

Gan, Z., Zhao, M.-B. and Chow, T. W. (2009b). Induction machine fault detection using clone selection programming, Expert Systems with Applications 36(4): 8000-8012.

Ghate, V. N. and Dudul, S. V. (2009). Fault diagnosis of three phase induction motor using neural network techniques, Proc. Second International Conference on Emerging Trends in Engineering 83 Technology (ICETET), number I, pp. 922-928.

Ghate, V. N. and Dudul, S. V. (2010). Optimal MLP neural network classifier for fault detection of three phase induction motor, Expert Systems with Applications 37(4): 3468-3481.

Ghate, V. N. and Dudul, S. V. (2011). Cascade neural network based fault classifier for three phase induction motor, IEEE Transactions on Industrial Electronics 58(5): 1555-1563.

Goldberg, P. E. (1989). Genetic Algorithm in Search Optimization and Machine Learning, 1st edn, Addison-Wesley Longman Publishing Co., New York.

Goode, P. (1995a). Using a neural/fuzzy system to extract heuristic knowledge of incipient faults in induction motors: Part II-Application, IEEE Transactions on Industrial Electronics 42(2): 139-146.

Goode, P. V. (1995b). Using a neural/fuzzy system to extract heuristic knowledge of incipient faults in induction motors. Part I-Methodology, IEEE Transactions on Industrial Electronics 42(2): 131-138.

Huang, X., Habetler, T. G. and Harley, R. G. (2007). Detection of rotor eccentricity faults in a closedloop drive-connected induction motor using an artificial neural network, IEEE Transactions on Power Electronics 22(4): 1552-1559.

Immovilli, F., Bellini, A., Rubini, R. and Tassoni, C. (2010). Diagnosis of bearing faults in induction machines by vibration or current signals: A critical comparison, IEEE Transactions on Industry Applications 46(4): 1350-1359.

Joksimovic, G. M. and Penman, J. (2000). The detection of inter-turn short circuits in the stator windings of operating motors, IEEE Transactions on Industrial Electronics 47(5): 1078-1084.
Kaminski, M., Kowalski, C. T. and Orlowska-Kowalska, T. (2010). General Regression Neural Networks as rotor fault detectors of the induction motor, Proc. IEEE International Conference on Industrial Technology (ICIT), pp. 1239-1244.

Kankar, P. K., Sharma, S. C. and Harsha, S. P. (2011). Fault diagnosis of ball bearings using machine learning methods, Expert Systems with Applications 38(3): 1876-1886.

Karakose, M., Aydin, I. and Akin, E. (2010). The intelligent fault diagnosis frameworks based on fuzzy integral, Proc. International Symposium on Power Electronics Electrical Drives Automation and Motion (SPEEDAM), pp. 1634-1639.

Karami, F., Poshtan, J. and Poshtan, M. (2010a). Broken bar fault detection in induction motors based on modified winding function, Proc. IEEE International Conference on Control Applications, pp. 1951-1956.

Karami, F., Poshtan, J. and Poshtan, M. (2010b). Detection of broken rotor bars in induction motors using nonlinear Kalman filters., ISA transactions 49(2): 189-95.

Kennedy, J. (1997). The particle swarm: social adaptation of knowledge, Proc. IEEE International Conference on Evolutionary Computation (ICEC'g'), pp. 303-308.

Kennedy, J. and Eberhart, R. (1995). Particle swarm optimization, Proc. International Conference on Neural Networks (ICNN'95), Vol. 3, pp. 1942-1948.

Kianinezhad, R., Nahid-Mobarakeh, B., Baghli, L., Betin, F. and Capolino, G.-A. (2008). Modeling and control of six-phase symmetrical induction machine under fault condition due to open phases, IEEE Transactions on Industrial Electronics 55(5): 19661977.

Kim, B., Lee, K., Yang, J., Lee, S. B., Wiedenbrug, E. J. and Shah, M. R. (2011). Automated detection of rotor faults for inverter-fed induction machines under standstill conditions, IEEE Transactions on Industry Applications 47(1): 55-64.

Kohler, J. L., Sottile, J. and Trutt, F. C. (1992). Alternatives for assessing the electrical integrity of induction motors, IEEE Transactions on Industry Applications 28(5): 1109-1117.

Kumar, K. V., Kumar, S. S., Praveena, B., John, J. P. and Paul, J. E. (2010). Soft computing based fault 
diagnosis, Proc. International Conference on Computing Communication and Networking Technologies (ICCCNT 2010), pp. 1-7.

Laurentys, C. A., Palhares, R. M. and Caminhas, W. M. (2010). Design of an artificial immune system based on Danger Model for fault detection, Expert Systems with Applications 37(7): 5145-5152.

Lee, S. B., Yang, J., Hong, J., Yoo, J., Kim, B., Lee, K., Yu, J., Kim, M., Lee, K., Wiedenbrug, E. J. and Nandi, S. (2011). A new strategy for condition monitoring of adjustable speed induction machine drive systems, IEEE Transactions on Power Electronics 26(2): 389-398.

Leite, D. F., Hell, M. B., Costa Jr., P. and Gomide, F. (2009). Real-time fault diagnosis of nonlinear systems, Nonlinear Analysis: Theory, Methods 8 Applications 71(12): e2665-e2673.

Li, B., Chow, M.-Y., Tipsuwan, Y. and Hung, J. C. (2000). Neural-network-based motor rolling bearing fault diagnosis, IEEE Transactions on Industrial Electronics 47(5): 1060-1069.

Mahamad, A. K. and Hiyama, T. (2008). Development of artificial neural network based fault diagnosis of induction motor dearing, Proc. IEEE 2nd International Power and Energy Conference (PECon'08), pp. 1387-1392.

Mahamad, A. K. and Hiyama, T. (2010). Fault classification performance of induction motor bearing using AI methods, Proc. 5th IEEE Conference on Industrial Electronics and Applications, pp. 56-61.

Marques Cardoso, A. J., Cruz, S. M. A. and Fonseca, D. S. B. (1999). Inter-turn stator winding fault diagnosis in three-phase induction motors, by Park's vector approach, IEEE Transactions on Energy Conversion 14(3): 595-598.

Martins, J. F., Pires, V. F. and Pires, A. J. (2007). Unsupervised neural-network-based algorithm for an on-line diagnosis of three-phase induction motor stator fault, IEEE Transactions on Industrial Electronics 54(1): 259-264.

Mini, V., Setty, S. and Ushakumari, S. (2010). Fault detection and diagnosis of an induction motor using fuzzy logic, Proc. IEEE Region 8 International Conference on Computational Technologies in Electrical and Electronics Engineering (SIBIRCON), number 3, pp. 459-464.
Nandi, S., Toliyat, H. A. and Li, X. (2005). Condition monitoring and fault diagnosis of electrical motors - A review, IEEE Transactions on Energy Conversion 20(4): 719-729.

Nemec, M., Drobnic, K., Nedeljkovic, D., Fiser, R. and Ambrozic, V. (2010). Detection of broken bars in induction motor through the analysis of supply voltage modulation, IEEE Transactions on Industrial Electronics 57(8): 2879-2888.

Onel, I. Y., Ayçiçek, E. and Senol, I. (2009). An experimental study, about detection of bearing defects in inverter fed small induction motors by Concordia transform, Journal of Intelligent Manufacturing 20(2): 243-247.

Ong, R., Dymond, J. and Findlay, R. (2000). Bearing damage analysis in a large oil-ring-lubricated induction machine, IEEE Transactions on Industrial Electronics 47(5): 1085-1091.

Pawlak, M. and Kowalski, C. (2010). Low-cost embedded system for the IM fault detection using neural networks, Proc. The XIX International Conference on Electrical Machines (ICEM 2010), pp. 1-5.

Pezzani, C. M., Donolo, P. D., Castellino, A. M., Bossio, G. R. and De Angelo, C. H. (2010). A new approach to the Park's vector for broken bars and load oscillation diagnosis on IM, Proc. IEEE International Conference on Industrial Technology (ICIT), pp. $1221-1226$.

Pires, D. F., Pires, V. F., Martins, J. F. and Pires, A. J. (2009). Rotor cage fault diagnosis in threephase induction motors based on a current and virtual flux approach, Energy Conversion and Management 50(4): 1026-1032.

Puche-Panadero, R., Pineda-Sanchez, M., Riera-Guasp, M., Roger-Folch, J., Hurtado-Perez, E. and PerezCruz, J. (2009). Improved resolution of the MCSA method via Hilbert transform, enabling the diagnosis of rotor asymmetries at very low slip, IEEE Transactions on Energy Conversion 24(1): 52-59.

Radhika, S., Sabareesh, G., Jagadanand, G. and Sugumaran, V. (2010). Precise wavelet for current signature in 3/IM, Expert Systems with Applications 37(1): 450-455.

Razik, H., Correa, M. B. A. D. R. and da Silva, E. R. C. (2009). A novel monitoring of load level and broken bar fault severity applied to squirrel-cage induction motors using a genetic algorithm, IEEE Transactions on Industrial Electronics 56(11): 4615-4626. 
Razik, H., Correa, M. B. R. and da Silva, E. R. C. (2010). The tracking of induction motor's faulty lines through particle swarm optimization using chaos, Proc. IEEE International Conference on Industrial Technology (ICIT), number 2, pp. 12451250 .

Riera-Guasp, M., Cabanas, M. F., Antonino-Daviu, J. a., Pineda-Sánchez, M. and García, C. H. R. (2010). Influence of nonconsecutive bar breakages in motor current signature analysis for the diagnosis of rotor faults in induction motors, IEEE Transactions on Energy Conversion 25(1): 80-89.

Rodriguez, P. V. J. and Arkkio, A. (2008). Detection of stator winding fault in induction motor using fuzzy logic, Applied Soft Computing 8(2): 1112-1120.

Romero-Troncoso, R. J., Saucedo-Gallaga, R., CabalYepez, E., Garcia-Perez, A., Osornio-Rios, R. A., Alvarez-Salas, R., Miranda-Vidales, H. and Huber, N. (2011). FPGA-based online detection of multiple combined faults in induction motors through information entropy and fuzzy inference. Aceito para publicação em 19 de Fevereiro.

Sadeghian, A., Ye, Z. and Wu, B. (2009). Online detection of broken rotor bars in induction motors by wavelet packet decomposition and artificial neural networks, IEEE Transactions on Instrumentation and Measurement 58(7): 2253-2263.

Samanta, B. and Nataraj, C. (2009). Use of particle swarm optimization for machinery fault detection, Engineering Applications of Artificial Intelligence 22(2): 308-316.

$\mathrm{Su}, \mathrm{H}$. and Chong, K. T. (2007). Induction machine condition monitoring using neural network modeling, IEEE Transactions on Industrial Electronics 54(1): 241-249.

Suetake, M., Da Silva, I. N. and Goedtel, A. (2010). Sistema fuzzy compacto embarcado em DSP e sua aplicação para controle $\mathrm{V} / \mathrm{F}$ de motores de indução, Controle \& Automação 21(3): 245-259.

Tallam, R. M., Habetler, T. G. and Harley, R. G. (2003). Stator winding turn-fault detection for closed-loop induction motor drives, IEEE Transactions on Industry Applications 39(3): 720-724.

Tallam, R. M., Lee, S. B., Stone, G. C., Kliman, G. B., Yoo, J., Habetler, T. G. and Harley, R. G. (2007). A survey of methods for detection of stator-related faults in induction machines, IEEE Transactions on Industry Applications 43(4): 920-933.
Tan, W. W. and Huo, H. (2005). A generic neurofuzzy model-based approach for detecting faults in induction motors, IEEE Transactions on Industrial Electronics 52(5): 1420-1427.

Thomson, W. T. and Fenger, M. (2001). Current signature analysis to detect induction motor faults, IEEE Industry Applications Magazine 7(4): 26-34.

Tran, V., Yang, B., Oh, M. and Tan, A. (2009). Fault diagnosis of induction motor based on decision trees and adaptive neuro-fuzzy inference, Expert Systems with Applications 36(2): 1840-1849.

Uddin, M. N., Wang, W. and Huang, Z. R. (2010). Modeling and minimization of speed ripple of a faulty induction motor with broken rotor bars, IEEE Transactions on Industry Applications 46(6): 22432250 .

Vas, P. (1993). Parameter Estimation, Condition Monitoring and Diagnosis of Electrical Machines, Clarendon Press, Oxford University Press, Oxford.

Vas, P. (1999). Artificial-Intelligent-Based Electrical Machines and Drives: Application of Fuzzy, Neural, Fuzzy-Neural and Genetic-Algorithm-Based Techniques, Oxford University Press, New York.

Wolbank, T. M., Nussbaumer, P., Chen, H. and Macheiner, P. E. (2011). Monitoring of rotor-bar defects in inverter-fed induction machines at zero load and speed, IEEE Transactions on Industrial Electronics 58(5): 1468-1478.

Wu, S. and Chow, T. (2004). Induction machine fault detection using SOM-based RBF neural networks, IEEE Transactions on Industrial Electronics 51(1): 183-194.

Xu, Z., Xuan, J., Shi, T., Wu, B. and Hu, Y. (2009). A novel fault diagnosis method of bearing based on improved fuzzy ARTMAP and modified distance discriminant technique, Expert Systems with Applications 36(9): 11801-11807.

Yan, J., Lu, L., Zhao, D. and Wang, G. (2010). Diagnosis of bearing incipient faults using fuzzy logic based methodology, Proc. Seventh International Conference on Fuzzy Systems and Knowledge Discovery, pp. 1229-1233.

Yang, D.-M. (2010). The application of artificial neural networks to the diagnosis of induction motor bearing condition using Hilbert-based bispectral analysis, Proc. 5th IEEE Conference on Industrial Electronics and Applications, number 2, pp. 1730-1735. 
Zadeh, L. (1965). Fuzzy sets, Information and Control 8(3): 338-353.

Zhang, L., Xiong, G., Liu, H., Zou, H. and Guo, W. (2010). Bearing fault diagnosis using multi-scale entropy and adaptive neuro-fuzzy inference, Expert Systems with Applications 37(8): 6077-6085.

Zidani, F., Diallo, D., Benbouzid, M. E. H. and NaitSaid, R. (2008). A fuzzy-based approach for the diagnosis of fault modes in a voltage-fed PWM inverter induction motor drive, IEEE Transactions on Industrial Electronics 55(2): 586-593.

Zidani, F., El Hachemi Benbouzid, M., Diallo, D. and Nait-Said, M. (2003). Induction motor stator faults diagnosis by a current concordia patternbased fuzzy decision system, IEEE Transactions on Energy Conversion 18(4): 469-475.

Zouzou, S., Laala, W., Guedidi, S. and Sahraoui, M. (2009). A fuzzy logic approach for the diagnosis of rotor faults in squirrel cage induction motors, Proc. Second International Conference on Computer and Electrical Engineering (ICCEE'09)), pp. 173-177. 